Bull. Austral. Math. Soc.

VOL. 54 (1996) [203-210]

\title{
HOMOGENEOUS POISSON STRUCTURES
}

\author{
F. Malek and A. Shafei Dem Abad
}

\begin{abstract}
In this paper we provide an algebraic definition for the Schouten product and give a decomposition for homogeneous Poisson structures in any $n$-dimensional vector space. A large class of $n$-homogeneous Poisson structures in $\mathbb{R}^{k}$ is also characterised.
\end{abstract}

\section{INTRODUCTION}

Poisson structures are among those mathematical structures which are most useful in mathematical physics. In many areas of physics and mechanics we encounter this type of structure in diverse forms. Even though there is much known about Poisson structures, there are still many unsolved important problems. One of these problems is that of classification, and another is the construction of Poisson structures. On manifolds, a Poisson structure is defined by a skew-symmetric contravariant 2-tensor whose Schouten product with itself is zero. Partial derivatives are involved in the expression for the Schouten product of two skew-symmetric contravariant tensors on manifolds. But for a large class of skew-symmetric contravariant tensors on $\mathbb{R}^{n}-$ those having polynomial coefficients in the standard basis - the Schouten product can be defined algebraically.

The algebraic definition of homogeneous Poisson structures of lower order namely constant, linear and quadratic - on vector spaces is already known. Constant Poisson structures are in fact skew-symmetric bilinear forms; linear Poisson structures on a vector space $E$ are in one-to-one correspondence with Lie algebra structures on $E^{*}$, and each quadratic Poisson structure on the vector space $E$ corresponds to a solution of the classical Yang-Baxter equation on End $\left(E^{*}\right)$, and vice versa [1].

In this paper, we first give an algebraic definition of general Poisson structures with polynomial coefficients on vector spaces, and then we give some results concerning homogeneous Poisson structures, and generalise to all homogeneous Poisson structures the decomposition of quadratic Poisson structures which has been given by Zhang-Ju Lie [4]. We also construct a large class of homogeneous Poisson structures on $\mathbb{R}^{n}$.

Received 24th October, 1995

This work is partially supported by the University of Tehran.

Copyright Clearance Centre, Inc. Serial-fee code: 0004-9729/96 \$A2.00+0.00. 


\section{Algebraic Approach to Polynomial Poisson Structures}

In the following, $H$ denotes an $n$-dimensional real vector space, $\left\{e_{i}\right\}_{i=1}^{n}$ is a basis for $H,\left\{x_{i}\right\}_{i=1}^{n}$ its corresponding coordinate functions.

Poisson structures whose bracket relations in terms of coordinates are polynomials are called polynomial Poisson structures. In the following (the $\mathbb{R}$-algebra) of antisymmetric contravariant tensors with polynomial coefficients on $H$ are called polynomial tensors (and will be denoted by $\Omega(H)$ ).

Let $\vee^{p}\left(H^{*}\right)$ and $\wedge^{q}(H)$ denote respectively the $p$-times symmetric tensor product of $H^{*}$ and the $q$-times exterior product of $H$, and let

$$
\vee\left(H^{*}\right) \otimes \wedge(H)=\oplus_{p, q} \vee^{p}\left(H^{*}\right) \otimes \wedge^{q}(H)
$$

For $\alpha \otimes v \in \vee^{p}\left(H^{*}\right) \otimes \wedge^{q}(H)$ and $\beta \otimes \omega \in \vee^{r}\left(H^{*}\right) \otimes \wedge^{s}(H)$, define

$$
(\alpha \otimes v) \cdot(\beta \otimes \omega)=\alpha \vee \beta \otimes v \wedge \omega \in \vee^{p+r}\left(H^{*}\right) \otimes \wedge^{q+r}(H)
$$

Under the above multiplication, $\vee\left(H^{*}\right) \otimes \wedge(H)$ becomes an associative $\mathbb{R}$-algebra. Let $\alpha \otimes v=\alpha^{1} \vee \cdots \vee \alpha^{n} \otimes v_{1} \wedge \cdots \wedge v_{m} \in \vee^{n}\left(H^{*}\right) \otimes \wedge^{m}(H)$. The linear operator

$$
D: \vee\left(H^{*}\right) \otimes \wedge(H) \rightarrow \vee\left(H^{*}\right) \otimes \wedge(H)
$$

is defined as follows:

$$
D(\alpha \otimes v)=\sum_{i, j=1}^{n, m}(-1)^{j+1} v_{j}\left(\alpha^{i}\right) \alpha^{1} \vee \cdots \vee \widehat{\alpha^{i}} \vee \cdots \vee \alpha^{n} \otimes v_{1} \wedge \cdots \wedge \widehat{v_{j}} \wedge \cdots \wedge v_{m}
$$

We say that $A=\sum \alpha_{i} \otimes v_{j}$ is exact if and only if $D(A)=0$.

There are some equivalent definitions of the Schouten product (see: $[2,3])$. The definition of the Schouten product which follows is an algebraic one equivalent to the others on $\Omega(H)$.

Definition 1.1: The Schouten product

$$
[\quad]: \vee\left(H^{*}\right) \otimes \wedge(H) \times \vee\left(H^{*}\right) \otimes \wedge(H) \rightarrow \vee\left(H^{*}\right) \otimes \wedge(H)
$$

is an $\mathbb{R}$-bilinear mapping defined by:

$$
\left[\begin{array}{ll}
U & V
\end{array}\right]=D(U \cdot V)-D(U) \cdot V-(-1)^{u} U . D(V)
$$

where $U \in \vee\left(H^{*}\right) \otimes \wedge^{u}(H)$ and $V \in \vee\left(H^{*}\right) \otimes \wedge(H)$ 
Now we are going to give a one-to-one correspondence between the polynomial Poisson structures on $H$ and some special structures on $\vee\left(H^{*}\right) \otimes \wedge(H)$.

Let $G=\sum_{i_{1}, \cdots, i_{n}} \sum_{j_{1}, \cdots, j_{m}} x^{i_{1}} x^{i_{2}} \cdots x^{i_{n}} e_{j_{1}} \wedge e_{j_{2}} \wedge \cdots \wedge e_{j_{m}} \in \Omega(H)$. The $\mathbb{R}$-algebra homomorphism $s: \Omega(H) \rightarrow \vee\left(H^{*}\right) \otimes \wedge(H)$ is defined by

$$
s(G)=\sum_{i_{1}, \cdots, i_{n}} \sum_{j_{1}, \cdots, j_{m}} x_{i_{1}} \vee x_{i_{2}} \vee \cdots \vee x_{i_{n}} \otimes e_{j_{1}} \wedge e_{j_{2}} \wedge \cdots \wedge e_{j_{m}} .
$$

It is clear that $s$ is an isomorphism. Its inverse will be denoted by $\triangle$.

LEMmA 1.1. Let $G \in \Omega(H)$, then $s \circ \nabla=D \circ s$, where $\nabla$ is the Koszul operator.

Let $p$ be an orientable $n$-manifold with a volume element $\Omega$. The element $\Omega$ induces an isomorphism $\Phi$ from the space of all $i$-multiple vector fields, $X^{i}(p)$, to the space of $(n-i)$-forms. Let $D$ denote the exterior derivative of differential forms. The Koszul operator $\nabla$ is defined by $\nabla=(-1)^{i+1} \Phi^{-1} \circ D \circ \Phi$, the pull-back of $D$ under the isomorphism $\Phi$. Now the definition of the Schouten product of $u \in X^{i}(p)$ and $v \in X^{j}(p)$ is given by

$$
\left[\begin{array}{ll}
u & v
\end{array}\right]=\nabla(u \wedge v)-\nabla(u) \wedge v-(-1)^{i} u \wedge \nabla(v)
$$

Proof: Let

$$
G=\sum r_{i_{1} \cdots i_{n}}^{j_{1} \cdots j_{m}} x^{i_{1}} x^{i_{2}} \cdots x^{i_{n}} e_{j_{1}} \wedge e_{j_{2}} \wedge \cdots \wedge e_{j_{m}} \in \Omega(H)
$$

then

$$
\nabla(G)=\sum r_{i_{1} \cdots i_{n}}^{j_{1} \cdots j_{m}} \sum_{s, k} \delta_{j_{k}}^{i_{s}}(-1)^{k+1} x^{i_{1}} x^{i_{2}} \cdots \widehat{x}^{i_{s}} \cdots x^{i_{n}} e_{j_{1}} \wedge e_{j_{2}} \wedge \cdots \wedge \widehat{e}_{j_{k}} \wedge \cdots \wedge e_{j_{m}},
$$

so

$$
\begin{aligned}
s \circ \nabla(G) & =\sum_{i_{1}, \cdots, i_{n}}^{j_{1}, \cdots, j_{m}} r_{i} \sum_{i_{s}=j_{k}}(-1)^{k+1} x^{i_{1}} x^{i_{2}} \cdots \widehat{x^{i}} \cdots x^{i_{n}} e_{j_{1}} \wedge e_{j_{2}} \wedge \cdots \wedge \widehat{e_{j_{k}}} \wedge \cdots \wedge e_{j_{m}} \\
& =D \circ s(G) .
\end{aligned}
$$

LEMMA 1.2 . Let $G \in \Omega(H)$. Then

$$
s\left[\begin{array}{ll}
G & G
\end{array}\right]=[s(G) s(G)]
$$

PROOF :

$$
\begin{aligned}
s\left[\begin{array}{l}
G \quad G
\end{array}\right] & =s(\nabla(G \wedge G)-2 \nabla(G) \wedge G) \\
& =s(\nabla(G \wedge G))-2 s(\nabla(G) \wedge G) \\
& =D(s(G) \cdot s(G))-2 D(s(G)) \cdot s(G) \\
& =[s(G) s(G)]
\end{aligned}
$$


Theorem 1.1. Let $P \in \vee\left(H^{*}\right) \otimes \wedge^{2}(H)$. Then $\triangle(P)$ is a polynomial Poisson structure on $H$ if and only if $\left[\begin{array}{ll}P & P\end{array}\right]=0$.

Proof: Let $\left[\begin{array}{ll}P & P\end{array}\right]=0$, then

$$
s[\triangle(P) \triangle(P)]=[s(\triangle(P)) s(\triangle(P))]=\left[\begin{array}{ll}
P & P
\end{array}\right]=0
$$

and therefore

$$
[\triangle(P) \triangle(P)]=0 .
$$

Conversely, suppose that $\triangle(P)$ is a polynomial Poisson structure on $H$; then $[\triangle(P) \triangle(P)]=0$. Therefore

$$
s([\triangle(P) \triangle(P)])=\left[\begin{array}{ll}
P & P
\end{array}\right]=0 .
$$

Remark. In [1] Bhaskara and Rama give a correspondence between quadratic Poisson structures on the vector space $H^{*}$ and solutions of the classical Yang-Baxter equation on the Lie algebra End $(H)$ as follows: Let $\left\{T_{i j}\right\}_{i, j=1}^{n}$ be the standard basis of End $(H)$. Assume that $\sum_{i, j, k, l=1}^{n} r_{i j}^{k l} T_{i j} \wedge T_{k l}$ is a solution of the classical Yang-Baxter equation on End $(H)$. If $\tilde{T}_{i j}=x_{j} \partial / \partial x_{i}$, then $\tilde{R}=\sum_{i, j, k, l=1}^{n} r_{i j}^{k l} \tilde{T}_{i j} \wedge \tilde{T}_{k l}$ is a quadratic Poisson structure. For more details see [1].

Since $R_{1}=T_{12} \wedge T_{34}$ and $R_{2}=T_{14} \wedge T_{32}$ give rise to the same quadratic Poisson structure $\widetilde{R}=\widetilde{T}_{12} \wedge \widetilde{T}_{34}=\widetilde{T}_{14} \wedge \widetilde{T}_{32}$, it is clear that this correspondence is not one-to-one. But the correspondence given in Theorem 1.1 is a one-to-one correspondence.

In what follows we shall use Theorem 1.1 implicity.

\section{Homogeneous Poisson Structures}

It is known that there exists a decomposition for any quadratic Poisson structure on a finite dimensional space [4]. Our next goal is to find out such a decomposition for all homogeneous Poisson structures.

LEMMA 2.1. Let $\pi=\sum_{i_{1}, \cdots, i_{k}} f_{i_{1} \cdots i_{k}} \partial / \partial x_{i_{1}} \wedge \partial / \partial x_{i_{2}} \wedge \cdots \wedge \partial / \partial x_{i_{k}}$ be an $n$ homogeneous contravariant $k$-tensor on the vector space $H$ and $I=\sum x_{j} \partial / \partial x_{j}$ be its identity vector field. Then $\left[\begin{array}{ll}I & \pi\end{array}\right]=(n-k) \pi$.

Proof: We can write:

$$
\left[\begin{array}{ll}
I & \pi
\end{array}\right]=\left[\sum_{j} x_{j} \partial / \partial x_{j} \sum_{i_{1}, \cdots, i_{k}} f_{i_{1} \cdots i_{k}} \partial / \partial x_{i_{1}} \wedge \partial / \partial x_{i_{2}} \wedge \cdots \wedge \partial / \partial x_{i_{k}}\right]
$$




$$
\begin{aligned}
& =\sum_{j, i_{1}, \cdots, i_{k}}\left(D\left(x_{j} f_{i_{1} \cdots i_{k}} \partial / \partial x_{j} \wedge \partial / \partial x_{i_{1}} \wedge \cdots \wedge \partial / \partial x_{i_{k}}\right)\right. \\
& \quad-D\left(x_{j} \partial / \partial x_{j}\right) \wedge f_{i_{1}, \cdots, i_{k}} \partial / \partial x_{i_{1}} \wedge \cdots \wedge \partial / \partial x_{i_{k}} \\
& \left.\quad+\left(x_{j} \partial / \partial x_{j}\right) \wedge D\left(f_{i_{1} \cdots i_{k}} \partial / \partial x_{i_{1}} \wedge \cdots \wedge \partial / \partial x_{i_{k}}\right)\right) \\
& =\sum x_{j} \partial f_{i_{1} i_{2} \cdots i_{k}} / \partial x_{j} \partial / \partial x_{i_{1}} \wedge \cdots \wedge \partial / \partial x_{i_{k}} \\
& \quad-f_{i_{1} \cdots i_{k}} \partial x_{j} / \partial x_{i_{1}} \partial / \partial x_{j} \wedge \partial / \partial x_{i_{2}} \wedge \cdots \wedge \partial / \partial x_{i_{k}} \\
& \quad+f_{i_{1} \cdots i_{k}} \partial x_{j} / \partial x_{i_{2}} \partial / \partial x_{j} \wedge \partial / \partial x_{i_{1}} \wedge \partial / \partial x_{i_{3}} \wedge \cdots \wedge \partial / \partial x_{i_{k}}+\cdots \\
& \quad+(-1)^{k} f_{i_{1} \cdots i_{k}} \partial x_{j} / \partial x_{i_{k}} \partial / \partial x_{j} \wedge \partial / \partial x_{i_{1}} \wedge \cdots \wedge \partial / \partial x_{i_{k-1}},
\end{aligned}
$$

which by the homogeneity of $f_{i_{1} i_{2} \cdots i_{k}}$ is equal to $n \pi-k \pi$.

Theorem 2.1. Any $n$-homogeneous Poisson structure on $\mathbb{R}^{k}$ has a unique decomposition $\pi=\pi_{X}+I \wedge X$, such that

$$
\left[\begin{array}{ll}
\pi_{X} & \pi_{X}
\end{array}\right]=-2(n-2) \pi \wedge X
$$

where $\pi_{X}$ is an exact 2-tensor, $I$ denotes the identity vector field and $X$ is an exact homogeneous vector field compatible with $\pi_{X}$, in the sense that $L_{X} \pi_{X}=0$.

Proof: By the above lemma

$$
\begin{aligned}
(n-2) \pi=\left[\begin{array}{ll}
I & \pi
\end{array}\right] & =D(I \wedge \pi)-D(I) \wedge \pi+I \wedge D(\pi) \\
& =D(I \wedge \pi)-k \pi+I \wedge D(\pi),
\end{aligned}
$$

so $\pi=1 /(n+k-2)[D(I \wedge \pi)+I \wedge D(\pi)]$. Let $\pi_{X}=1 /(n+k-2) D(I \wedge \pi)$ and $X=1 /(n+k-2) D(\pi)$. Then $\pi_{X}=\pi+X \wedge I$, and so

$$
\begin{aligned}
& {\left[\begin{array}{ll}
\pi_{X} & \pi_{X}
\end{array}\right]=[\pi+X \wedge I \quad \pi+X \wedge I]} \\
& =\left[\begin{array}{ll}
\pi & \pi
\end{array}\right]+2\left[\begin{array}{ll}
\pi & X \wedge I]+[X \wedge I \quad X \wedge I]
\end{array}\right]
\end{aligned}
$$

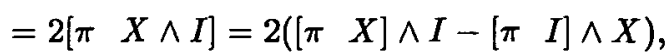

and since $\left[\begin{array}{ll}\pi & X\end{array}\right]=\left[\begin{array}{ll}\pi & D(\pi)\end{array}\right]=0$, then $\left[\begin{array}{ll}\pi_{X} & \pi_{X}\end{array}\right]=-2\left[\begin{array}{ll}\pi & I\end{array}\right] \wedge X=-2(n-2) \pi \wedge X$. On the other hand,

$$
\begin{aligned}
& {\left[\begin{array}{ll}
\pi_{X} & X
\end{array}\right]=\left[\begin{array}{ll}
\pi+X \wedge I & X
\end{array}\right]=\left[\begin{array}{ll}
\pi & X
\end{array}\right]+\left[\begin{array}{lll}
X \wedge I & X
\end{array}\right]} \\
& =\left[\begin{array}{ll}
X \wedge I & X
\end{array}\right]=\left(\left[\begin{array}{ll}
I & X
\end{array}\right] \wedge X-\left[\begin{array}{ll}
X & X
\end{array}\right] \wedge I\right) \\
& =\left[\begin{array}{ll}
I & X
\end{array}\right] \wedge X=(n-1) X \wedge X=0 .
\end{aligned}
$$

This shows that $\pi_{X}$ and $X$ are compatible with each other. 
To prove that the above decomposition is unique, suppose that $\pi$ has another decomposition

$$
\pi=\pi_{Y}-Y \wedge I
$$

then

$$
\pi_{Y}-Y \wedge I=\pi_{X}-X \wedge I
$$

so

$$
\pi_{Y}-\pi_{X}=(Y-X) \wedge I
$$

The left-hand side of the above equality is an exact tensor but the following lemma shows that the right-hand side is not exact unless $(Y-X) \wedge I=0$. Therefore $\pi_{Y}=\pi_{X}$.

LEMMA 2.2. Let $Y$ be an n-homogeneous vector field on $\mathbb{R}^{k}$. Then $Y \wedge I$ is equal to zero or it is not an exact tensor.

Proof: Let $Y \wedge I$ be an exact tensor. Then

$$
\begin{aligned}
(n-1) Y=\left[\begin{array}{ll}
I & Y
\end{array}\right] & =D(I \wedge Y)-D(I) \wedge Y+I \wedge D(Y) \\
& =D(I \wedge Y)-k Y+I \wedge D(Y)
\end{aligned}
$$

so

$$
(n+k-1) Y=D(I \wedge Y)+I \wedge D(Y)=I \wedge D(Y)
$$

giving

$$
Y=1 /(n+k-1) I \wedge D(Y)
$$

consequently

$$
Y \wedge I=1 /(n+k-1) I \wedge D(Y) \wedge I=0 .
$$

From the above theorem we obtain that:

Corollary 2.1. Any $n$-homogeneous Poisson structure $\pi$ on $\mathbb{R}^{k}$ has a decompositition $\pi=\pi_{X}-X \wedge I$. If $n=2$ or $k=3, \pi_{X}$ is an exact Poisson structure.

Proof: By the last theorem

$$
\left[\begin{array}{ll}
\pi_{X} & \pi_{X}
\end{array}\right]=-2(n-2) \pi \wedge X
$$

thus if $n=2,\left[\begin{array}{ll}\pi_{X} & \pi_{X}\end{array}\right]=0$. Let $k=3$. Then

$$
\left[\begin{array}{ll}
\pi & \pi
\end{array}\right]=D(\pi \wedge \pi)-2 D(\pi) \wedge \pi=0 .
$$

On the other hand $\pi \wedge \pi$ is a 4-tensor in $\mathbb{R}^{3}$ and so $\pi \wedge \pi=0$. Therefore $D(\pi) \wedge \pi=$ $X \wedge \pi=0$.

The next theorem gives us a large class of $n$-homogeneous Poisson structures in $\mathbb{R}^{k}$. First note the following evident proposition: 
Proposition 2.1. Let $X$ be an exact homogeneous vector field. Assume that there exists an exact linear vector field $Y$ such that $L_{X} Y=0$. Then $X \wedge Y=\pi_{X}$ is an exact homogeneous Poisson structure and $\pi_{X}+X \wedge I$ is a homogeneous Poisson structure.

THEOREM 2.2. Let $X$ be a homogeneous vector field in $\mathbb{R}^{k}$. Then $X \wedge I$ is a homogeneous Poisson structure in $\mathbb{R}^{k}$.

Proof: It is evident that $X \wedge I=D(X \wedge I) \wedge I$, so in the above proposition it suffices to put $Y=0$.

Corollary 2.2. For every two $n$-homogeneous vector fields $X$ and $Y$ the Poisson structures $X \wedge I$ and $Y \wedge I$ are compatible with each other.

Proof: Observe that

$$
X \wedge I+Y \wedge I=(X+Y) \wedge I
$$

clearly $X+Y$ is an $n$-homogeneous vector field, and by the last theorem $(X+Y) \wedge I$ is a Poisson structure.

The following proposition shows that the class of all $n$-homogeneous Poisson structures of the form $X \wedge I, X \in X_{p}\left(\mathbb{R}^{k}\right)$, is maximal. Let $X_{p}\left(\mathbb{R}^{k}\right)$ denote the set of polynomial vector fields on $\mathbb{R}^{k}$ homogeneous of degree $p$.

Proposition 2.2. If an $n$-homogeneous Poisson structure $\pi$ is compatible with $X \wedge I$ for all $X$ in $X_{p}\left(\mathbb{R}^{k}\right)$, then there exists $Y$ in $X_{p}\left(\mathbb{R}^{k}\right)$ such that $\pi=Y \wedge I$.

Proof: Let $\pi$ be an $n$-homogeneous Poisson structure on $\mathbb{R}^{k}$ which is compatible with each $X \wedge I, X \in X_{p}\left(\mathbb{R}^{k}\right)$. Then there exists a unique exact 2-tensor $\pi_{Y}$ and a homogeneous vector field $Y$ such that

$$
\pi=\pi_{Y}+Y \wedge I \text {. }
$$

Let $X$ be in $X_{p}\left(\mathbb{R}^{k}\right)$. Then

$$
\begin{aligned}
0 & =\left[\begin{array}{ll}
\pi & X \wedge I
\end{array}\right]=\left[\pi_{Y}+Y \wedge I \quad X \wedge I\right]=\left[\begin{array}{ll}
\pi_{Y} & X \wedge I
\end{array}\right] \\
& =\left[\begin{array}{ll}
\pi_{Y} & X
\end{array}\right] \wedge I-X \wedge\left[\begin{array}{ll}
\pi_{Y} & I
\end{array}\right]=\left[\begin{array}{ll}
\pi_{Y} & X
\end{array}\right] \wedge I-(n-2) X \wedge \pi_{Y}
\end{aligned}
$$

so

$$
\left[\pi_{Y} \quad X\right] \wedge I=(n-2) X \wedge \pi_{Y}
$$

or

$$
\left[\pi_{Y} \quad X\right] \wedge I \wedge I=(n-2) X \wedge \pi_{Y} \wedge I=0 .
$$

Since $X$ is an arbitrary element of $X_{p}\left(\mathbb{R}^{k}\right)$, then $\pi_{Y} \wedge I=0$. On the other hand

$$
(n-2) \pi_{Y}=\left[\begin{array}{ll}
\pi_{Y} & I
\end{array}\right]=D\left(\pi_{Y} \wedge I\right)-D\left(\pi_{Y}\right) \wedge I-\pi_{Y} \wedge D(I)=-k \pi_{Y} .
$$

This shows that $\pi_{Y}=0$. Therefore $\pi=Y \wedge I$, since $-k \neq n-2$. 


\section{REFERENCES}

[1] K.H. Bhaskara and K. Rama, 'Quadratic Poisson structures', J. Math. Phys. 32 (1991), 2314-2322.

[2] K.H. Bhaskara and K. Viswanath, Poisson algebra and Poisson manifolds, Pitman Research Notes in Mathematics 174 (Longman Science and Technology, Harlow, 1988).

[3] J-L. Koszul, 'Corochets de Schouten Nijenhuiset Cohomologie', Asterisque Soc. Math. de France (1985), 257-271.

[4] Lie Zhang-Ju, 'On quadraic Poisson structures', Lett. Math. Phys. 26 (1992), 33-42.

Department of Mathematics

The University of Tehran

Tehran

Iran

e-mail: malfer@irearn.bitnet

\author{
Institute for Studies in \\ Theoretical Physics and Mathematics \\ IPM \\ Tehran \\ Iran \\ and \\ Department of Mathematics \\ Faculty of Sciences \\ University of Tehran \\ Tehran \\ Iran
}

